\title{
PARQUE NACIONAL BERNARDO O'HIGGINS/TERRITORIO KAWÉSQAR WAES: CONSERVACIÓN Y GESTIÓN EN UN TERRITORIO ANCESTRAL
}

\author{
JUAN-CARLOS ARAVENA ${ }^{a}$, GERMAYNEE VELA-RUIZ ${ }^{\mathrm{b}}$, JULIANA TORRES ${ }^{\mathrm{b}}$, \\ CAROLINA HUENUCOYc \& JUAN-CARLOS TONKOc
}

\section{RESUMEN}

La gestión de áreas protegidas y la conservación de sus recursos naturales es una materia en permanente desarrollo y sobre la cual se han planteado diversos enfoques. Actualmente excluir a las comunidades humanas con derechos ancestrales de los parques nacionales es políticamente inviable y percibido como éticamente injustificable, sobre todo en países en desarrollo donde es cada vez más importante el reconocimiento de los derechos de sus habitantes. Es por ello, que los enfoques preservacionistas han ido cambiando hacia enfoques cooperativos y participativos, donde la expectativa es que el ser humano y su actuar sean consistentes con los objetivos de conservación de las áreas protegidas, sin dejar de lado las necesidades de quienes habitan dichos territorios.

En este trabajo presentamos la experiencia de planificación del Parque Nacional Bernardo O'Higgins (PBNO), el más grande del país. En el marco de un proyecto de investigación que buscaba definir una línea base de los recursos naturales de este parque, identificar las áreas con distinta vocación de uso, y el potencial económico asociado a actividades de turismo al interior del parque, incorporaron los intereses del pueblo originario involucrado ancestralmente con los territorios protegidos, logrando expresar estos intereses en los resultados obtenidos.

En el desarrollo de esta experiencia se generaron resultados no esperados que trascendieron los objetivos originales del proyecto: i) Se revisaron los antecedentes de la zonificación y uso del borde costero del territorio al interior del PNBO, de modo de influir en las definiciones respecto del desarrollo de actividades de acuicultura al interior del Parque. ii) La definición de los fiordos y canales interiores como parte de la superficie protegida en el Parque Nacional, lo que en la práctica supone incorporar una gran área marina protegida. iii) La participación de la comunidad Kawésqar con derechos ancestrales sobre el territorio en todo el proceso de planificación, desde la recolección de la información de terreno, pasando por la definición de las zonas de uso, y la discusión de las implicancias en el manejo de tan extensa área protegida.

PALABRAS CLAVE: Territorio Kawésqar, Conservación, Manejo, áreas protegidas.

a Universidad de Magallanes, Av. Bulnes 01855 Punta Arenas, Chile. $\$ juan.aravena@umag.cl

b Centro Regional Fundación CEQUA, Av. España 184. Punta Arenas, Chile. germaynee.velaruiz@cequa.cl, juliana.torres@cequa.cl

c Comunidad Kawésqar de Puerto Edén, Puerto Edén s/n, Chile. huenucoy@gmail.com, juancarlostonko@gmail.com 


\title{
BERNARDO O'HIGGINS NATIONAL PARK/ KAWÉSQAR WAES LAND: CONSERVATION AND MANAGEMENT IN AN ANCESTRAL LAND
}

\begin{abstract}
The management of protected areas and the conservation of its natural resources is a matter continuously discussed and subjected to different approaches. Today excluding people from national parks is politically infeasible and ethically unjustifiable, moreover, recognition of humans rights living in or adjacent to protected areas have also been increasing. Therefore, approaches have changed from a preservationist to cooperative and participatory; especially in developing countries, where the human being and their activities should be consistent with conservation objectives, without ignoring the needs of those who live within or adjacent to these territories.

In this paper we present our experience developing the management plan of the Bernardo O'Higgins National Park (BONP), the largest in our country. Within the research work, the basal line of natural resources of this national park, the different uses zones and the economic potential associated with touristic activities were defined. The local communities' goals, of those who have been historically involved with the protected area were incorporated, resulting in a management plan that includes in a more participatory way the local community.

During the development of this project we achieve non-expected results that exceeded our original objectives: i) we confronted the established use of the regional's coastline areas forbidding aquiculture activities within the BONP limits. ii) Defined fiords and channels as part of the protected area which would enlarge the National Park and transform it into a marine protected area. iii) The participation of the Puerto Eden's Kawesqar community in all the BONP planning process, from the field data collection, through the use zones definition, and the discussion of the management plan of the protected area.
\end{abstract}

KEY WORDS: Kawésqar territory, Conservation, Management, protected areas.

\section{INTRODUCCIÓN}

Las relaciones entre gestión de recursos naturales, especialmente aquellos situados en áreas protegidas y el desarrollo económico de territorios con una alta riqueza en patrimonio natural y cultural, plantea desafíos y oportunidades. Cada proyecto de desarrollo lleva aparejado una tensión inevitable entre el resguardo de este patrimonio y el uso de los recursos, ya se trate de proyectos de explotación directa como es el caso de proyectos extractivos, o el uso indirecto como en el caso de los proyectos turísticos.

Tradicionalmente la conservación de la naturaleza en países en vías de desarrollo como Chile ha incluido una escasa participación de las comunidades locales. Esto se refleja en que gran parte de los territorios protegidos bajo el Sistema Nacional de Áreas Silvestres Protegidas del Estado (SNASPE) fueran definidos en base a decisiones estatales, estableciéndose mayormente en territorios con baja densidad poblacional, bajo valor comercial y con escaso interés de privados (Pauchard \& Villarroel, 2002). Sin embargo, de acuerdo a Aylwin (2011) un 90\% de la superficie protegida por el SNASPE correspondería a territorios ancestrales indígenas. En consecuencia, la protección de la naturaleza bajo este enfoque implicó que a lo largo del siglo XX muchas comunidades indígenas fueran expulsadas de sus territorios ancestrales. En general, la gestión de las áreas protegidas en Chile se ha realizado sin la participación de las comunidades aledañas ni indígenas, y sin insertar dichos territorios en el contexto de desarrollo provincial y regional (Fuentes \& Domínguez, 2011). Como consecuencia de lo anterior, las experiencias de participación de pueblos originarios se restringen a convenios 
de colaboración y desarrollo de concesiones turísticas por parte de comunidades indígenas (Aylwin, 2011). Esta situación dista mucho aún de llevar a acuerdos de co-manejo, que permitirian integrar realmente en la toma de decisiones sobre la conservación de la naturaleza a los pueblos originarios.

En este trabajo presentamos un caso de estudio que vincula temáticas tales como la gestión de áreas silvestres protegidas, la participación de pueblos originarios, el desarrollo territorial, la investigación científica y políticas públicas respecto de los recursos naturales. La experiencia incluyó el desarrollo de una línea base de los recursos naturales y culturales del área silvestre protegida más grande de Chile: el Parque Nacional Bernardo O'Higgins (PNBO). La información recopilada y procesada en la línea base fue aplicada en el diseño de un plan de manejo y un plan de desarrollo turístico para el parque. Este proceso de investigación y planificación fue realizado gracias al proyecto "Caracterización Territorial del Parque Nacional Bernardo O'Higgins: su potencial económico, turístico, científico y cultural", mandatado por la Corporación Nacional Forestal (CONAF) Región de Magallanes y Antártica Chilena. Dicho proyecto fue ejecutado por el Centro Regional Fundación CEQUA y contó con el apoyo financiero del programa estatal INNOVA - CORFO. Este proyecto contó con la activa participación a lo largo de todo el proyecto de la Comunidad Indígena Kawésqar Residente en Puerto Edén, última comunidad sociológica de esta etnia y reconocidos como Tesoro Humano Vivo por la UNESCO en el año 2009 (Consejo Nacional de la Cultura y las Artes, 2012).

ÁREA DE ESTUDIO:

PARQUE NACIONAL BERNARDO O'HIGGINS/TERRITORIO KAWÉSQAR WAES

El PNBO es el área silvestre protegida con mayor superficie del Hemisferio Sur, con 4.475.905 hectáreas, de las cuales cerca de 3,8 millones de hectáreas corresponden a superficie terrestre y 750 mil hectáreas a superficie marina (Fig. 1). El parque comprende las áreas continentales y de archipiélagos que se extienden entre las latitudes $47^{\circ} 55^{\prime} S$ y $51^{\circ} 37^{\prime}$ S, un área ubicada en las regiones de Aysén y Magallanes, las más australes de Chile, incluyendo todo el territorio chileno de Campo de Hielo Sur (1.300.000 ha). Su administración está a cargo de la Corporación Nacional Forestal (CONAF) dependiente del Ministerio de Agricultura.

El PNBO se destaca por los siguientes atributos:

i) Es un área con más de 3.8 millones de hectáreas terrestres protegidas, más la totalidad de canales y fiordos incluidos en este territorio (750 mil hectáreas), siendo una de las áreas menos alteradas por actividades humanas. La diversidad de fiordos, canales, glaciares del Campo de Hielo Sur, islas, montañas y costas expuestas al Pacífico, sustenta una gran riqueza de recursos bióticos, culturales y paisajísticos.

ii) $\quad \mathrm{El} \mathrm{PNBO}$ se encuentra incluido completamente en el territorio ancestral del pueblo Kawésqar o Kawésqar Wæes, el cual se extiende desde el Golfo de Penas hasta el Estrecho de Magallanes (Fig. 1). Las evidencias de uso humano en el área se remontan a usos culturales ancestrales por parte de los Kawésqar de al menos $4.520 \pm 60$ años atrás (Legoupil \& Sellier, 2004), quienes se distinguía como un pueblo cazador-recolector, que mantuvo un modo de vida trashumante hasta hace unas pocas décadas atrás, manteniendo un bajo impacto sobre el entorno. Los escasos descendientes puros de la etnia Kawésqar aún viven dentro de los límites del Parque, resguardando su conocimiento por medio de su lengua nativa y la tradición oral, así como algunos usos y tradiciones que aún pueden llevar a cabo.

iii) $\quad$ El PNBO es un testimonio de los diversos procesos geológicos, glaciológicos y biológicos que han ocurrido en la Tierra durante los últimos 2,6 millones de años de historia geológica (periodo conocido como Cuaternario), y que hoy están estrechamente vinculados a la presencia y acción de una de las mayores áreas englaciadas del planeta: el Campo de Hielo Sur. Éste representa un laboratorio natural idóneo para la 


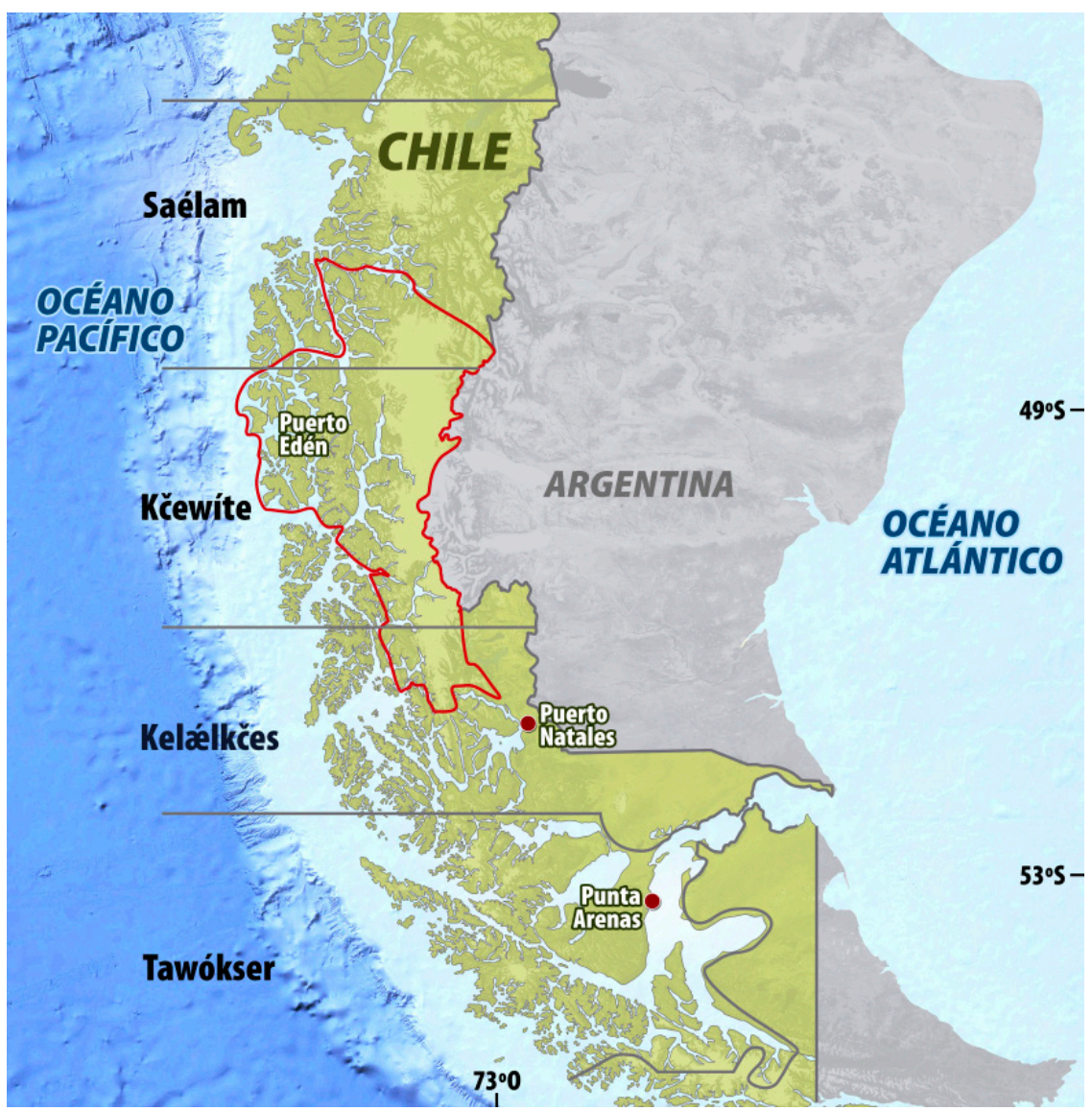

Fig. 1. Mapa del Parque Nacional Bernardo O'Higgins, indicando los límites del parque (línea roja), así como la división del territorio ancestral reconocida por el pueblo Kawésqar (líneas grises horizontales). En la figura se nombran las cuatro divisiones territoriales identificadas por Aguilera (2008). Fuente: Elaboración propia.

investigación de la relación entre los cambios que operan en el clima y los glaciares, una reserva estratégica de agua dulce y un recurso turístico de gran interés.

\section{METODOLOGÍA}

El proyecto se desarrolló entre los años 2009 y 2011, y contempló el desarrollo de tres etapas consecutivas: 1) Generación de línea base, 2) Etapa de planificación estratégica y zonificación, y 3) Análisis del potencial turístico del Parque Nacional Bernardo O'Higgins. Todas estas etapas se realizaron bajo un enfoque de involucramiento de la Comunidad Indígena Kawésqar Residente en Puerto Edén, así como de CONAF Magallanes y Antártica Chilena, institución a cargo de la administración del área protegida, generando una triangulación de actores trabajando colaborativamente, tal y como muestra la Fig. 2, y que se puede convertir en un ejemplo de desarrollo de proyectos en áreas silvestres protegidas en territorio ancestral de pueblos originarios:

\section{Etapa de generación de} información de línea base

En el PNBO la línea base incluyó tanto una revisión bibliográfica, como un exhaustivo trabajo científico de terreno. En el levantamiento de información trabajaron más de 50 especialistas, que participaron en 7 excursiones de una duración promedio de 20 días cada una, lo que significó alrededor de 6 meses de trabajo de terreno. 


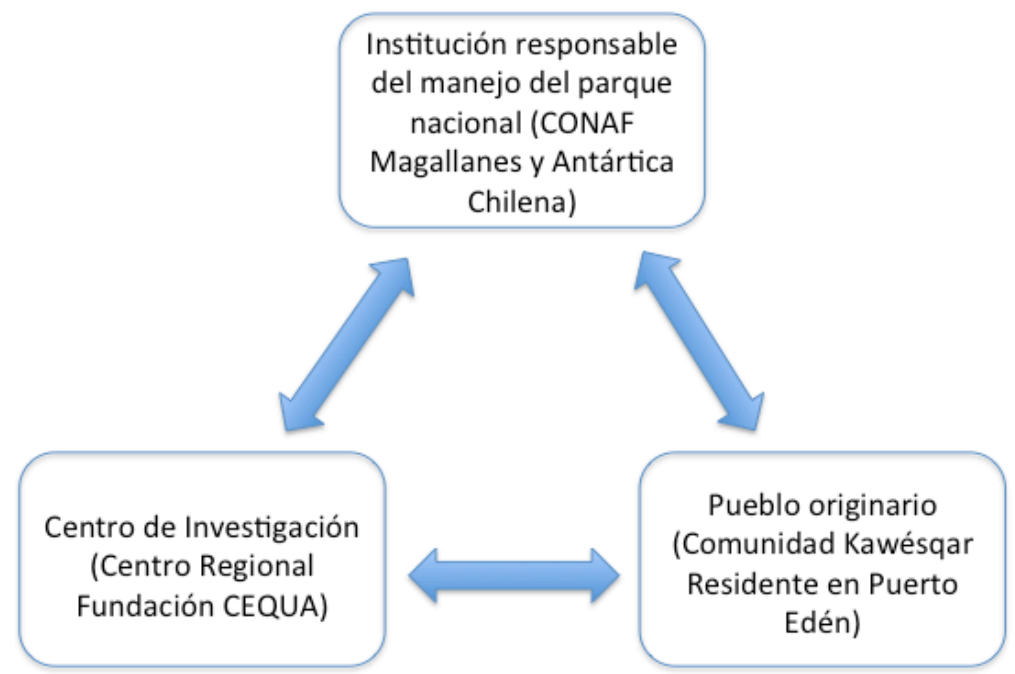

Fig. 2. Actores participantes del proceso de planificación del Parque Nacional Bernardo O'Higgins: comunidad indígena -administrador del área protegida- academia.

Los especialistas incluyeron una amplia gama de disciplinas, que consideraron estudios sobre flora terrestre, mamíferos marinos y terrestres, aves marinas y terrestres, huemules, roedores, anfibios, insectos, bentos, peces, parámetros oceanográficos, geomorfología, paisaje, ecología, etnogeografía, arqueología y turismo.

Las excursiones de terreno incluyeron 60 días del grupo de flora y fauna terrestre (noviembre de 2009 y enero de 2010), 60 días del grupo de biodiversidad marina (enero a marzo 2010), 25 días del grupo de arqueología (2009), 20 días del grupo de etnogeografía (2010) y 7 días del grupo de turismo (2010).

\section{Planificación estratégica del área protegida}

Uno de los objetivos más importantes del proyecto "Parque Nacional Bernardo O'Higgins: su potencial económico, turístico, científico y cultural" fue la realización del plan de manejo como un documento directriz de gestión para el Parque. La definición del plan de manejo según lo planteado para el SNASPE es "un instrumento de gestión, que se fundamenta en un proceso de planificación y que comprende aspectos técnicos, normativos y orientadores destinados a garantizar la conservación de un área protegida, a través del ordenamiento del uso de su espacio" (Núñez, 2008).
Para el caso del PNBO, la metodología de planificación de áreas protegidas propuesta por Núñez (2008) fue adaptada al contexto del Parque y complementada por diversas actividades que mejoran la implementación de la metodología propuesta. Las modificaciones a la metodología de CONAF corresponden a una elaboración de una línea base del parque debido a que no existía información de terreno suficiente sobre esta área protegida. Además, se amplió la participación de comunidades aledañas e insertas en el parque, realizándose talleres de planificación participativa en Puerto Edén, Puerto Natales y Punta Arenas de la Región de Magallanes; y en Caleta Tortel, Villa O'Higgins y Coyhaique de la Región de Aysén. Así mismo, destaca la activa participación de la comunidad indígena Kawésqar de Puerto Edén a lo largo de las etapas de levantamiento de información base, planificación y potencial turístico a través de reuniones, talleres y visitas a terreno. Todo lo anterior permitió generar un plan de manejo acorde al contexto, la realidad y las necesidades encontradas en el territorio.

Análisis del potencial turístico

El análisis del potencial turístico del PNBO se desarrolló durante la última fase del proyecto, utilizando como insumo central la línea base generada y el plan de manejo del PNBO, lo cual 
fue complementado con varias actividades que permitieron obtener resultados más representativos. Estas actividades incluyeron:

- Excursión de terreno de prospección del potencial turístico con expertos y representantes de instituciones públicas.

- Sistematización de información de oferta y demanda turística: la información de oferta y demanda turística se identificó a partir de la recopilación de estudios, informes técnicos, estadísticas de instituciones públicas y empresas vinculadas al turismo regional, autoridades $y$ sitios de Internet.

- Realización de encuestas a operadores turísticos: se aplicaron encuestas a operadores turísticos de la región con respecto a sus intereses respecto del PNBO. Dicha información permitió recopilar datos de primera mano para completar las estadísticas de oferta turística.

- Taller de expertos: en diciembre de 2010 se realizó un taller de expertos participantes en la línea base del primer año del proyecto. En éste participaron funcionarios de CONAF, investigadores de CEQUA y de otras instituciones, miembros de la Comunidad Indígena Kawésqar, funcionarios de servicios públicos, consultores. Esta actividad tenía como objetivo lograr una ponderación de la importancia de los elementos consignados en las investigaciones de la línea base del PNBO para su uso en la elaboración del plan de manejo del parque. Esta sistematización sirvió además como insumo para la evaluación de los aspectos de historia natural y de riqueza cultural en la jerarquización de los sitios con potencial turístico del parque.

La vastedad del territorio del PNBO hizo necesario llevar a cabo un proceso de selección donde se evaluaron los atractivos con potencial turístico basado en los atributos paisajísticos, científicos, culturales y territoriales, empleando para ello la metodología de evaluación del patrimonio turístico desarrollada por el Centro Interamericano de Capacitación Turística y la Organización de los Estados americanos (CICATUR/OEA, 1983). Esta metodología permitió generar una clasificación que facilitó una evaluación objetiva, pero a ello se sumó al criterio empírico de operadores y empresarios turísticos, experiencia y recomendaciones de la Armada de Chile, más las consideraciones aportadas por la comunidad indígena Kawésqar de Puerto Edén.

\section{RESULTADOS}

\section{Linea base}

El estudio permitió realizar una línea base a macroescala en los más de 3,5 millones de hectáreas terrestres y de las 750 mil hectáreas marinas contenidas en el parque nacional. Debido a la gran cantidad de información generada, el análisis de los resultados ha dado lugar a informes técnicos, presentaciones a congresos científicos, publicaciones, y productos de difusión que se espera continúen conforme se avance en los análisis resultantes de los recursos naturales y culturales del Parque. En la Tabla 1 se enumeran algunos de los productos de divulgación de los resultados de la línea base del PNBO.

\section{Zonificación y plan de manejo}

Los resultados de la aplicación de la metodología permitieron generar una zonificación y un plan de manejo para el Parque Nacional Bernardo O'Higgins. Los aspectos que resaltan de este proceso son:

- Inclusión de la variable cultural compuesta por la arqueología y la etnogeografía, asociada a la participación de la Comunidad Kawésqar de Puerto Edén como criterio relevante en la zonificación. Esta información se ha vuelto fundamental para poner en valor el patrimonio cultural de este parque nacional.

- Inclusión de las comunidades aledañas al PNBO, Comunidad Kawésqar de Puerto Edén, instituciones públicas con injerencia, guardaparques y expertos en talleres desarrollado a lo largo de todo el proceso de planificación. 
Tabla 1. Productos de difusión generados a partir de la línea base del Parque Nacional Bernardo O'Higgins.

\begin{tabular}{|c|c|c|c|}
\hline \# & Referencia & Tipo de producto & Descripción \\
\hline 1 & CEQUA, 2011a & Informe técnico & $\begin{array}{l}\text { Informe Final del Proyecto Innova Corfo 08CTU01-20 } \\
\text { (proyecto PNBO). }\end{array}$ \\
\hline 2 & $\begin{array}{l}\text { Vela-Ruiz \& Aravena, } \\
2011\end{array}$ & Informe técnico & $\begin{array}{l}\text { Análisis de la propuesta de zonificación del Borde Costero } \\
\text { para la región de Magallanes en base a los resultados del } \\
\text { proyecto PNBO. }\end{array}$ \\
\hline 3 & CEQUA, $2011 b$ & Libro de difusión & $\begin{array}{l}\text { Guía bilingüe (español/inglés) con los principales resultados } \\
\text { del proyecto } \mathrm{PNBO} \text {. }\end{array}$ \\
\hline 4 & $\begin{array}{l}\text { Aguilera \& Tonko, } \\
2011 .\end{array}$ & Libro de difusión & $\begin{array}{l}\text { Guía bilingüe (español/kawésqar) con los resultados de la } \\
\text { investigación etnogeográfica del proyecto PNBO. }\end{array}$ \\
\hline 5 & Vela-Ruiz et al. 2011 & Libro de difusión & $\begin{array}{l}\text { Guía con el análisis de las posibilidades de desarrollo } \\
\text { turístico en el PNBO. }\end{array}$ \\
\hline 6 & Guzmán, 2010 & $\begin{array}{l}\text { Artículo en revista } \\
\text { científica. }\end{array}$ & $\begin{array}{l}\text { Publicación con los resultados del muestreo de micro- } \\
\text { roedores del PNBO. }\end{array}$ \\
\hline 7 & Marín et al. 2013 & $\begin{array}{l}\text { Artículo en revista } \\
\text { científica. }\end{array}$ & $\begin{array}{l}\text { Publicación incluyendo los resultados del muestreo de } \\
\text { huemules en el PNBO. }\end{array}$ \\
\hline 8 & Vela-Ruiz et al. 2013 & $\begin{array}{l}\text { Artículo en revista } \\
\text { científica. }\end{array}$ & $\begin{array}{l}\text { Publicación incluyendo el análisis del potencial turístico del } \\
\text { PNBO. }\end{array}$ \\
\hline 9 & Domínguez et al. 2015 & Capítulo de libro. & $\begin{array}{l}\text { Publicación con los resultados del muestreo de la vegetación } \\
\text { de turberas en el PNBO. }\end{array}$ \\
\hline 10 & $\begin{array}{l}\text { Jerez \& Muñoz- } \\
\text { Escobar, } 2015\end{array}$ & Capítulo de libro. & $\begin{array}{l}\text { Publicación incluyendo los resultados de insectos asociados } \\
\text { a la vegetación de turberas en el PNBO. }\end{array}$ \\
\hline 11 & Ortiz, 2015 & Capítulo de libro. & $\begin{array}{l}\text { Publicación incluyendo los resultados de anfibios asociados } \\
\text { a la vegetación de turberas en el PNBO. }\end{array}$ \\
\hline 12 & Riveros et al. 2015 & Capítulo de libro. & $\begin{array}{l}\text { Publicación incluyendo los resultados de avifauna asociada a } \\
\text { la vegetación de turberas del PNBO. }\end{array}$ \\
\hline 14 & Guzmán, 2015 & Capítulo de libro. & $\begin{array}{l}\text { Publicación incluyendo los resultados de micro-roedores } \\
\text { asociados a la vegetación de turberas del PNBO. }\end{array}$ \\
\hline 15 & Esquete \& Aldea, 2015 & $\begin{array}{l}\text { Artículo en revista } \\
\text { científica. }\end{array}$ & $\begin{array}{l}\text { Publicación reportando la descripción de una nueva especie } \\
\text { de invertebrado marino en el PNBO. }\end{array}$ \\
\hline
\end{tabular}

- Definición del área de influencia ecológica, socioeconómica y política del PNBO. Generación de una zonificación que incluye la porción terrestre y marina, así como divide el territorio del PNBO en zonas de uso: cultural, intangible, primitivo, en recuperación y uso público.

- Plan de manejo que establece la zonificación y planificación estratégica del PNBO al mediano y largo plazo.

La zonificación propuesta y el plan de manejo resultantes fueron entregados como productos del informe final del proyecto INNOVA-
CORFO en octubre del 2011. El plan de manejo ha pasado por diversas etapas de trabajo por parte de CONAF Magallanes, CONAF Aysén y CONAF nacional entre los años 2011 y 2018, debido a que cambios políticos, administrativos y metodológicos han hecho necesario actualizar el plan de manejo a las necesidades actuales. En estos años la participación de la Comunidad Indígena Kawésqar Residente en Puerto Edén ha sido permanente y coherente con el espíritu del Convenio 169 de la OIT y Tratados internacionales que protegen sus derechos ancestrales. Se espera que la oficialización del nuevo plan de manejo sea ratificado durante el año 2018. 


\section{Análisis del potencial turístico}

El trabajo desarrollado permitió definir ocho sectores en los cuales se presentan no sólo las áreas marítimas, sino su conexión con áreas terrestres. Éstas últimas pueden complementar e innovar las actividades turísticas en el parque, ampliando el uso marítimo y potenciando el nuevo conocimiento que se ha obtenido sobre su flora y vegetación, fauna terrestre, biodiversidad marina, arqueología y etnogeografía, paleontología y patrimonio geológico entre otros, dándole paso a un turismo más complementario hasta el hoy encontrado en el territorio. El resultado de la evaluación del potencial turístico del PNBO permitió definir que los atractivos turísticos centrales en esta área protegida son los glaciares y fiordos, así como la fauna marina asociada. Son estos atractivos en definitiva los que motivan mayoritariamente la llegada de turistas al Parque, los cuales son potenciados por los atractivos complementarios que provienen de los atributos científicos y culturales asociados a cada área del PNBO.

Los sectores que presentan mayor potencial para el desarrollo turístico según la metodología aplicada son en orden de importancia: 1) Puerto Edén y alrededores, 2) fiordo Eyre y glaciar Pío XI, 3) glaciar O'Higgins, 4) monte Balmaceda, 5) glaciar
Jorge Montt, 6) fiordos Peel - Amalia - Asia y Calvo, 7) fiordo Témpano y 8) fiordo Europa. En la Tabla 2 se presentan los sectores identificados como de mayor potencial para el desarrollo turístico en el PNBO, indicando el principal atractivo asociado.

\section{Resultados no esperados}

Fuera de los resultados referidos a los objetivos iniciales del proyecto de caracterización del Parque Nacional Bernardo O'Higgins, en el desarrollo del proyecto nos encontramos con temáticas inicialmente no consideradas y de gran relevancia para la región. A continuación describimos algunas de ellas.

Zonificación del borde costero: instalación de Áreas Apropiadas para la Acuicultura (AAA)

El 3 de febrero de 2011 la Comisión Regional de Uso del Borde Costero (CRUBC) aprobó la Propuesta de Zonificación del Borde Costero para la Provincia de Última Esperanza (UE), provincia en la cual se ubican casi tres cuartas partes de la superficie terrestre total del PNBO. Esta aprobación fue parte del proceso de más de cuatro años durante el que la CRUBC definió zonas de uso preferente para el borde costero de

Tabla 2. Sectores con potencial turístico en el PNBO y su atractivo principal.

\begin{tabular}{|c|c|c|}
\hline Jerarquía & Sector & Principal Atractivo \\
\hline 1 & Puerto Edén & $\begin{array}{l}\text { Comunidad Indígena Kawésqar, su cultura y su entorno. Atractivo del poblado, sitio de interés } \\
\text { geológico. Posibilidad de complementar circuitos en el PNBO. Posibilidad de conectar por } \\
\text { sendero de trekking con el glaciar Pío XI. }\end{array}$ \\
\hline 2 & $\begin{array}{l}\text { Fiordo Eyre y } \\
\text { glaciar Pío XI }\end{array}$ & $\begin{array}{l}\text { Avance del glaciar Pio XI, uno de los pocos glaciares del campo de hielo sur que aún avanza. } \\
\text { Sector de atractivo geológico, vegetación terrestre y de fauna marina. Uno de los mayores } \\
\text { atractivos paisajísticos. }\end{array}$ \\
\hline 3 & Glaciar O'Higgins & $\begin{array}{l}\text { Navegación en el lago complementado con trekking en el Campo de Hielo Sur. Cercanía a } \\
\text { ciudad argentina del Chaltén, conexión con el Parque Nacional los Glaciares de Argentina. }\end{array}$ \\
\hline 4 & Monte Balmaceda & $\begin{array}{l}\text { Integración de los parques nacionales Torres del Paine y Bernardo O־Higgins, con actividades } \\
\text { enfocadas en un turismo de naturaleza. Cercanía a la ciudad de Puerto Natales. }\end{array}$ \\
\hline 5 & $\begin{array}{l}\text { Glaciar Jorge } \\
\text { Montt }\end{array}$ & $\begin{array}{l}\text { Glaciar con mayor retroceso en el Campo de Hielo Sur. Cercanía a Campo de Hielo Norte } \\
\text { y Carretera Austral, posibilidad de integrar circuitos turísticos. }\end{array}$ \\
\hline 6 & $\begin{array}{l}\text { Fiordos Peel - } \\
\text { Amalia -Asia y } \\
\text { Calvo }\end{array}$ & $\begin{array}{l}\text { Sector con alta densidad de glaciares complementados con circuitos terrestres en valles con } \\
\text { importante presencia de huemules y fauna marina. }\end{array}$ \\
\hline 7 & Fiordo Témpano & $\begin{array}{l}\text { Sector con la población de huemul con mayor densidad del país hasta el momento conocida. } \\
\text { Asociado a proyecto nacional de conservación del huemul y potencial turismo científico. }\end{array}$ \\
\hline 8 & Fiordo Europa & Observación de glaciar de Campo de Hielo Sur y de fauna marina \\
\hline
\end{tabular}




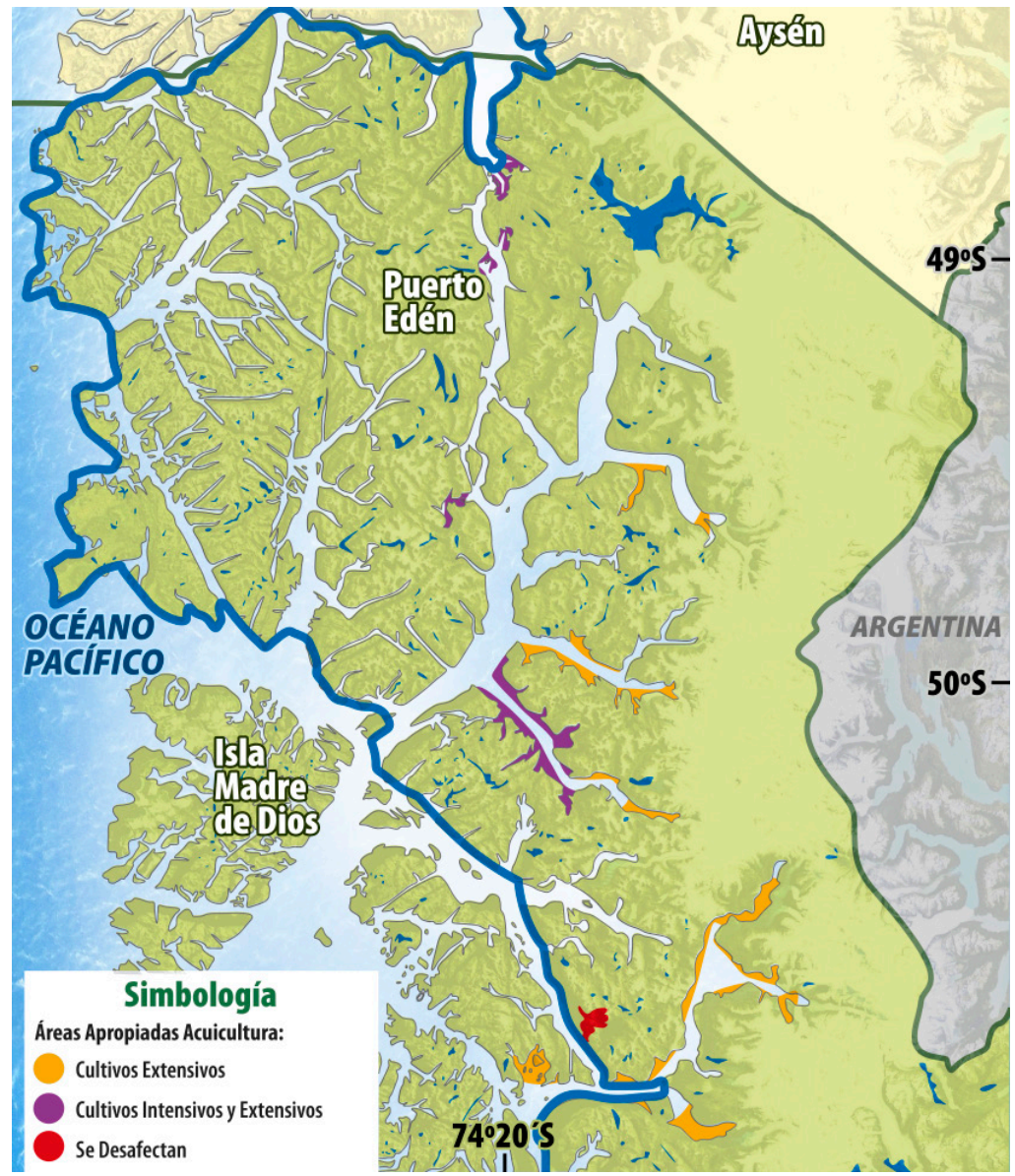

Fig. 3. Áreas al interior de Parque Nacional Bernardo O'Higgins incluidas en la propuesta de zonificación del Borde Costero de la Región de Magallanes y Antártica Chilena al año 2011. _Límite Parque;__Límite Región; __ Límite Internacional. Fuente: Adaptación propia a partir de GORE Magallanes, 2011.

la Región de Magallanes. En la Fig. 3 se muestra la propuesta de Áreas Apropiadas para la Acuicultura (AAA) insertas en el PNBO diferenciando entre aquellas que serían destinadas a cultivos extensivos (principalmente mitílidos, indicadas en color verde), y las que se usarían para cultivos tanto extensivos como intensivos (mitílidos y salmonicultura, respectivamente; indicadas en color púrpura).

Como resultado de un desfase entre el proceso de zonificación de borde costero referido $y$ el estudio de línea base marina y terrestre del PNBO y su entorno, la información del proyecto Innova PNBO no alcanzó a ser considerada en las discusiones finales del proceso de zonificación realizadasainiciosdelaño2011. Comoestasituación implicaba no considerar importantes antecedentes recabados durante el desarrollo del proyecto, se elaboró un informe técnico que confrontaba la zonificación aprobada con estos antecedentes (Vela-Ruiz \& Aravena, 2011). En este informe se planteó la paradoja de que el Estado Chileno, en su ineludible y urgente tarea de planificar el uso de sus recursos naturales costeros, defina usos contrapuestos con estudios financiados por el mismo Estado para determinar la potencialidad de usos de bajo impacto para sus áreas silvestres protegidas. Además entra en vigencia el Convenio 169 de la Organización Internacional del Trabajo (OIT) sobre pueblos indígenas y tribales en países independientes el 15 de septiembre de 2009. Este tratado internacional reconoce derechos sobre territorios ancestralmente reconocidos, en el uso 
y disfrute de recursos naturales que en ellos se encuentran, a desarrollar su propia cultura material e inmaterial, ser consultados sobre la base del consentimiento libre, previo e informado mediante procedimientos apropiados cada vez que prevean medidas susceptibles de afectarles directamente. Así mismo los Estados deberán tomar medidas, en conjunto con la comunidad indígena, para proteger y preservar el medio ambiente de los territorios que habitan ancestralmente (Biblioteca del Congreso Nacional, 2009).

En el informe de Vela-Ruiz \& Aravena (2011) se hizo ver que la propuesta de borde costero para el PNBO se hizo sin considerar los antecedentes que el proyecto Innova para este parque recopiló en los últimos dos años. Dichos antecedentes dijeron relación con las siguientes temáticas:

- Paisaje: La valoración de la calidad y fragilidad visual de las unidades de paisaje analizadas en el PNBO dieron como resultados que en todos los casos el criterio de riesgo de uso público a considerar fuera alto o muy alto. Esto hace recomendable que las intervenciones sean de tipo muy moderado para no alterar las condiciones de gran valor paisajístico que posee este parque. La definición de algunas de las zonas más vulnerables de este punto de vista como áreas apropiadas para la acuicultura corresponde a una evidente contradicción.

- Turismo: Los antecedentes entregados dan cuenta que varias de las zonas definidas como aptas para la acuicultura tienen un potencial mucho mayor para actividades turísticas; más aún, algunas de ellas ya están siendo utilizadas con este propósito.

- Biodiversidad: La creación misma del PNBO tuvo como objetivo central la preservación de la gran riqueza de biodiversidad de sus ecosistemas. El estudio de línea base realizado por el proyecto Innova Corfo confirmó esta riqueza e impone la obligación de hacer un correcto uso de las herramientas de planificación territorial para cuidar de este patrimonio natural. La zona oriental de canales y fiordos continentales es una macrozona de características ecológicas únicas, dado el evidente efecto que tiene el aporte de agua dulce que recibe del Campo de Hielo Sur.
Las comunidades bentónicas asociadas a los canales y fiordos continentales en el PNBO constituyen una situación ecológica con muy pocos correlatos en el resto del planeta.

- Patrimonio cultural: Junto con la gran riqueza de biodiversidad del $\mathrm{PNBO}$, el patrimonio cultural que representa el pueblo Kawésqar $y$ sus derechos ancestrales son centrales en cualquier definición o planificación en este territorio. La consideración de estos elementos ha enriquecido enormemente el mencionado proyecto y plantean desafíos innovadores en el trato de las relaciones de los pueblos originarios y las áreas silvestres protegidas.

- Patrimonio Geológico: La consideración de este aspecto en la valoración de los ecosistemas del PNBO es una aproximación innovadora que ha tenido un desarrollo reciente. De ser una categoría descriptiva pasa a ser una variable que puede poner de relieve valores que es necesario proteger.

\section{Participación Kawésqar}

Históricamente, las participación e incorporación de las comunidades indígenas en los procesos de definición de gestión y administración de las áreas protegidas son escasos y muchas veces no consideradas, dejando fuera a éstas en la toma de decisiones transcendentales en el manejo y conservación en sus propios territorios ancestrales. Para la Comunidad Indígena Kawésqar Residente en Puerto Edén la preservación del $\mathrm{PNBO}$ es un compromiso que tiene que ver con su propia identidad como pueblo indígena nómadacazador-recolector. Este conocimiento ancestral de un territorio tan extenso es lo que permitió beneficiar las diferentes etapas del proyecto aquí descrito, y reconocer formalmente, la importancia de esta comunidad indígena en la conservación y manejo del PNBO. Es por esto, que este proyecto sentó precedentes de trabajo colaborativo donde el conocimiento científico en conjunto con el conocimiento ancestral son mecanismos que permiten establecer un diálogo constructivo para lograr objetivos de conservación.

Lo anterior, sin embargo, se da en un contexto político y legal contrastante. Por un lado, los tratados internacionales ratificados por el Estado 
de Chile, entre los que se encuentran el Declaración de las Naciones Unidas sobre los Derechos de los Pueblos Indígenas (Naciones Unidas, 2008), Convenio 169 de la Organización Internacional del Trabajo (Biblioteca del Congreso Nacional de Chile, 2009), el Pacto Internacional de Derechos Civiles y Políticos (Biblioteca del Congreso Nacional de Chile, 1989a), y el Pacto Internacional de Derechos Económicos, Sociales y Culturales (Biblioteca del Congreso Nacional de Chile, 1989b), reconocen el derecho de los pueblos originarios y de las minorías étnicas a desarrollar su propia cultura material e inmaterial en su territorio ancestral. Por otro lado, el año 2008 se promulgó la política de espacios costeros para pueblos originarios, la cual, aunque distribuye los espacios costeros de acuerdo al uso que determine la comunidad indígena, no consideró del todo aspectos culturales ancestrales. Sumado a lo anterior, la crisis sanitaria de la industrial salmonicultura en la Región de los Lagos, llevó a esta industria a demandar áreas aptas para la acuicultura en la Región de Magallanes, decisión aprobada por el Estado sin consulta previa, libre e informada a la Comunidad Indígena Kawésqar Residente en Puerto Edén. Todos estos aspectos incidieron en llevar a cabo la formalización de las dimensiones del territorio ancestral de esta comunidad indígena en enero de 2013 en el Primer Congreso Kawésqar realizado en el sector de Jetarkte, en lo que se conoce como la Declaración de Jetarkte. (http://comunidadkawesqar-puertoeden.blogspot.cl/2013/01/ declaracion-de-jetarkte.html). En esta declaración se reconoce que el Parque Nacional Bernardo O'Higgins en su totalidad es parte importante del territorio ancestral de la Comunidad Kawésqar Residente en Puerto Edén (Kawésqar wæs).

Considerando que actualmente la administración de las áreas silvestre protegidas del Estado recae en la Corporación Nacional Forestal, la probable entrada en vigencia del plan de manejo en el año 2018 y las dimensiones de protección del medio marino y terrestre del Parque Nacional Bernardo O'Higgins que hasta el día de hoy carece con protección efectiva, hará necesario que CONAF y la Comunidad Indígena Kawésqar Residente en Puerto Edén logren generar acuerdos conducentes a la gobernanza en conjunto del $\mathrm{PNBO}$. Aspectos relevantes de este acuerdo serán considerar al medio marino dentro de su diseño, rescatar la cultura material, promover y proteger la cultura inmaterial, y preservar el territorio ancestral kawésqar.

\section{Gestión territorial}

Puerto Edén, poblado ubicado a 260 millas náuticas de navegación al norte de Puerto Natales es considerada una de las localidades más aisladas de Chile y la más cercana a Campo de Hielo Sur. Sin embargo, pese a ser una zona de la mayor importancia según diferentes indicadores, esta zona evidencia una compleja precariedad. Esta situación fue señalada por los habitantes de Puerto Edén dentro de los talleres de trabajo de construcción del plan de manejo, y que tienen un fuerte componente de dependencia de recursos naturales al interior del área protegida. Esta situación se produce aun cuando han existido iniciativas de inversión por parte del Estado, con una escuela dotada de buenos servicios e infraestructura, pero que atendía a sólo siete estudiantes; un muelle con modernas instalaciones pero que es claramente subutilizado; una antena de comunicación que permite una muy buena conexión por telefonía celular e internet, pero que atiende a una población cada vez menor. Matus (2008) describió la precariedad de Puerto Edén en relato sobre el efecto devastador de la aparición de la marea roja en estas latitudes a partir del año 1994 y como los intentos del Estado de Chile por solucionar una crisis por momentos terminal, no ha impedido que el resultado sea poco esperanzador. La población local ha desarrollado sus medios de vida basado en la pesca artesanal, contando con un bajo capital social y humano que les dificultan aprovechar las oportunidades que el turismo genera en esta área silvestre protegida (Vela-Ruiz, 2009). Estas brechas se mantienen al día de hoy, a pesar de que la temporada 2016 - 2017 entró en operación un nuevo ferry subvencionado por el Estado que une Puerto Natales - Puerto Edén y Caleta Tortel, el cual permite el aumento de visitantes en la localidad, pero sin considerar preparar la infraestructura y el capital humano para la llegada de visitantes. Así, Puerto Edén siendo el único poblado al oeste del Campo de Hielo Sur, con potenciales de naturaleza prístina innegables, continúa en una situación de desamparo evidente, 
representando una de nuestras fronteras interiores más precarias.

\section{DISCUSIÓN}

A partir de una propuesta científica que buscaba aplicar una metodología estándar en la prospección de recursos naturales (línea base), en la formulación de un plan de manejo, y en el análisis del potencial turístico de un área protegida, se abrieron una variedad de temas conectados y con una importante repercusión en materias de orden socioeconómico, de políticas públicas y de manejo de recursos naturales. Especialmente en la fase de talleres de discusión se desbordaron aspectos que conllevaban la contraposición de intereses, perspectivas y modelos de desarrollo en ocasiones totalmente contrapuestos.

Una primera reflexión surgida de nuestra experiencia elaborando un plan de manejo para un parque nacional tan vasto como el $\mathrm{PNBO}$, es la necesidad de conciliar la estandarización del método usado en las distintas Áreas Silvestres Protegidas de Estado (ASPE) con las particularidades de cada región, en particular en lo que dice relación con la participación de las comunidades indígenas en el manejo de las mismas áreas protegidas. En el ejemplo del PNBO, la incorporación de la Comunidad Kawésqar Residente en Puerto Edén enriqueció todo el proceso, relevando el patrimonio cultural de esta área protegida y aportando antecedentes que han permitido correlacionar los resultados científicos con los empíricos de esta comunidad indígena. De esta forma el proyecto sentó precedentes a nivel nacional sobre la participación real de las comunidades indígenas de acuerdo a sus derechos ancestrales y en reconocimiento del Convenio 169 de la OIT, más allá de los procedimientos establecidos en el Reglamento que regula el procedimiento de consulta indígena (Decreto 66/2014 del Ministerio de Desarrollo Social). Este respaldo científico para la Comunidad Indígena Kawésqar Residente en Puerto Edén en el PNBO ha permitido visibilizarlo y ser reconocido como una comunidad fundamental para el manejo y conservación de esta área silvestre protegida. Esta recomendación es pertinente para la nueva metodología de planificación de las áreas protegidas, basada en el método de "Estándares
Abiertos" implementada desde el año 2017 por CONAF (CONAF, 2017), ya que desde los aprendizajes del proyecto se demuestra que es posible establecer una relación positiva entre la academia, la comunidades indígenas, comunidad local y la entidad responsable de la administración del área protegida, que permite relevar los aspectos de interés. Los nuevos desafíos que surgen desde esta iniciativa es cómo generar acuerdos de gobernanza entre las comunidades indígenas y el Estado de Chile, representado a través de CONAF en este caso, que permitan co-manejar las ASPE ubicadas en territorios ancestrales indígenas.

Esta experiencia de participación de la Comunidad Indígena Kawésqar de Puerto Edén en el levantamiento de información de base y planificación de un área protegida, actualmente es un referente para los procesos de consulta indígena, una manera de avanzar y demostrar que las preservación del medio natural y cultural de las áreas silvestres protegidas en Chile no es freno para su desarrollo, es una forma de asegurar la conservación de estas áreas silvestres para las generaciones actuales y futuras, y también para toda la humanidad.

En otro sentido, siendo los planes de manejo importantes instrumentos de gestión, sin una apropiada provisión de recursos financieros para implementar las necesidades de los programas definidos, estos documentos se transforman en meros ejercicios teóricos sin capacidad de concretar estas necesidades en acciones fecundas y de acuerdo con la realidad local. La falta de inversión que refuerce los objetivos de conservación, investigación y divulgación en los actuales parques nacionales en Chile en general es una carencia que atenta contra su cabal desarrollo. Esta carencia como se ha visto en el ejemplo del PNBO rebasa el sólo aspecto de protección que representan los parques y tiene connotaciones hacia el desarrollo socioeconómico y cultural de las regiones en que se ubican. En definitiva se trata de evitar el generar áreas protegidas de papel y que éstos representen buenos balances entre los diferentes usos que buscan promover.

Una segunda reflexión son los desafíos que genera el haber reconocido los fiordos y canales como parte del Parque Nacional Bernardo O'Higgins. Esto además de beneficiar 
la conservación, ya que implica la prohibición del desarrollo de la acuicultura en su interior, plantea enormes desafíos para un área donde el medio terrestre y el medio marino son una misma unidad de protección y de gestión efectiva. Hoy en día, esta realidad no ha sido enfrentada adecuadamente por el Estado Chileno, ya que la protección marina no es responsabilidad de la Corporación Nacional Forestal, por lo tanto no cuenta con los recursos financieros ni técnicos adecuados para manejar efectivamente 750 mil hectáreas. Esta realidad se complejiza aún más, cuando históricamente se ha desarrollado la pesca artesanal dentro del parque nacional, actividades que no son compatibles por definición, pero que requieren enfrentarse y lograr trabajar de forma colaborativa.

Una tercera reflexión que aflora de esta visión que excede los objetivos de nuestro proyecto es la preocupante tendencia al despoblamiento de la única localidad habitada de la Patagonia occidental chilena, Puerto Edén. Situación compleja, ya que el desarrollo de este poblado está estrechamente ligado al uso consuntivo de recursos naturales, incluida la extracción de madera para construcción y calefacción, así como para la sustentación de actividades productivas como la pesca artesanal. Innegablemente, Puerto Edén se configura como el mayor punto de acceso a los atributos turísticos que tiene el Parque Nacional Bernardo O'Higgins, por lo tanto, es la mayor oportunidad de desarrollo que tiene la localidad considerando la categoría de parque nacional que tiene el territorio y el medio marino que la rodea. Sin embargo, requiere de una fuerte inversión y apoyo gubernamental, ya que los habitantes tienen una identidad territorial vinculada a la pesca artesanal y no al turismo, así como debilidades en su capital humano y organización social, lo que dificultan los procesos de diversificación productiva (Vela-Ruiz, 2009).

Finalmente, luego de 6 años de finalizado el proyecto Innova Corfo, ha habido mínimos avances desde la institucionalidad pública, existiendo aún importantes desafíos de gestión efectiva de este territorio que supera los 4 millones de ha. y que debe lograr congeniar las visiones y el manejo entre las regiones de Aysén y Magallanes. Se requiere mantener los esfuerzos de investigación y conservación en este importante territorio de fiordos y canales, el cual representa ecosistemas de alto valor para la conservación a nivel nacional $e$ internacional.

\section{AGRADECIMIENTOS}

Los autores agradecen el financiamiento del proyecto Innova Corfo "Caracterización Territorial del Parque Nacional Bernardo O'Higgins: potencial económico, turístico, científico y cultural" Cód. 08CTU01-20, así como al Centro Regional Fundación CEQUA, los investigadores, profesionales y otras instituciones que participaron en el referido proyecto. Agradecemos todo el trabajo desarrollado por los miembros de la Comunidad Kawésqar Residente en Puerto Edén, en especial al invaluable conocimiento de la Sra. Gabriela Paterito y del Sr. Francisco Arroyo, así como el trabajo desarrollado por José Tonko, Oscar Aguilera, María Isabel Tonko y todos los integrantes de la comunidad que participaron del proyecto. Agradecemos la participación y compromiso de Rafael Contreras funcionario de CONAF Provincia de Última Esperanza, y de los profesionales de la Dirección Regional de CONAF Magallanes y Antártica Chilena Mauricio Ruiz, Alejandra Silva y Michael Arcos, quiénes fueron parte de un proceso participativo que ha sentado precedentes en el trabajo con pueblos originarios, centros de investigación y áreas silvestres protegidas.

\section{BIBLIOGRAFÍA}

Aguilera, O. (2008). Escenario en los relatos de viaje Kawésqar. Onomázein, 18(2), 49-74.

Aguilera, O., \& Tonko, J. (2011). Guía etnogeográfica del Parque Nacional Bernardo O'Higgins. Punta Arenas: Ediciones CEQUA.

Aylwin, J. (2011). Conservación en territorios indígenas: Marcos jurídicos y experiencias nacionales y comparadas $y$ directrices internacionales. En J. Aylwin \& X. Cuadra (Eds.), Los Desafíos de la Conservación en los Territorios Indigenas en Chile. (pp. 9-92). Temuco: Observatorio de Derechos de los Pueblos Indígenas.

Biblioteca del Congreso Nacional de Chile. (1989a). Decreto 778. Promulga el Pacto Internacional de Derechos Civiles y Políticos adoptado por la Asamblea General de la Organización de las Naciones Unidas por Resolucion $n^{\circ} 2.200$, el 16 de diciembre de 1966 y 
suscrito por Chile en esa misma fecha. Recuperado de https://www.leychile.cl/Navegar?idNorma=15551

Biblioteca del Congreso Nacional de Chile. (1989b). Decreto 326. Promulga el Pacto Internacional de Derechos Económicos, Sociales y Culturales. Adoptado por la Asamblea General de la Organización de las Naciones Unidas el 19 de diciembre de 1966, suscrito por Chile el 16 de septiembre de 1969. Recuperado de https://www.leychile.cl/Navegar?idNorma=12382

Biblioteca del Congreso Nacional de Chile. (2009). Decreto 236. Promulga el Convenio $N^{\circ} 169$ sobre pueblos indígenas y tribales en países independientes de la Organización Internacional del Trabajo. Recuperadode https://www.leychile.cl/Navegar?idNorma=279441

Consejo Nacional de la Cultura y las Artes. (2012). Tesoros Humanos Vivos. Patrimonio Cultural Inmaterial. Santiago: Publicaciones Cultura. Recuperado de: http:// www.cultura.gob.cl/wp-content/uploads/2012/03/ THV.pdf.

CEQUA (2011a). Informe Final. Proyecto Innova Corfo. "Caracterización territorial del Parque Nacional Bernardo O'Higgins: potencial turístico, económico, científico y cultural. Punta Arenas, Chile, Fundación CEQUA.

CEQUA (2011b). Parque Nacional Bernardo O'Higgins National Park. Punta Arenas: Ediciones CEQUA.

CICATUR/OEA (1983). Vejsbjerg, L., Calvo, J., Heredia, S. (2018). Criterios de Valoración Turística-Recreativa de Recursos Geológicos, Paleontológicos y Paisajísticos. Caso Monumento Natural Parque de los Dinosaurios. Ciudad de Neuquén.

CONAF. (2017). Manual para la planificación del manejo de las áreas protegidas del SNASPE. Santiago, Corporación Nacional Forestal de Chile.

Domínguez, E., Vega-Valdés, D., Dollenz, O., Villa-Martínez, R., Aravena, J. C., Henríquez, J. M., \& Muñoz-Escobar, C. (2015). Flora y vegetación de turberas en la región de Magallanes. En E. Domínguez \& D. Vega-Vádes (Eds.), Funciones y servicios ecosistémicos de las turberas en Magallanes. Colección Libros INIA $N^{\circ} 33$ (pp. 149-195). Punta Arenas, Instituto de Investigaciones Agropecuarias, Centro Regional de Investigación Kampenaike.

Esquete, P., \& Aldea, C. (2015). Leucothoe kawesqari, a new amphipod from Bernardo O'Higgins National Park, with remarks on the genus in the Magellan region (Crustacea, Peracarida). ZooKeys, 539, 85-96.

Fuentes, E., \& Domínguez, R. (2011). Aplicación y resultados de la encuesta sobre efectividad de manejo de las principales áreas protegidas de Chile, Proyecto GEFPNUD-MMA "Creación de un Sistema Nacional Integral de Áreas Protegidas para Chile”. Santiago, Ministerio del Medio Ambiente.

Gore Magallanes (2011). Estrategia Regional de Desarrollo de Magallanes 2012-2020. Gobierno Regional de Magallanes y Antártica Chilena.

Guzmán, J. (2010). Mamalia, Rodentia, Sigmodontinae, Abrothrix lanosus (Thomas 1897): topotype, distribution and new locality records for Chile. Check List, 6(4), 383-386.

Guzmán, J. (2015). Roedores de las turberas en Magallanes. En E. Domínguez y D.Vega-Valdés (Eds.), Funciones y servicios ecosistémicos de las turberas en Magallanes, Colección Libros INIA $N^{\circ} 33$ (pp.279-293). Punta Arenas, Instituto de Investigaciones Agropecuarias. Centro Regional de Investigación Kampenaike.

Jerez, V., \& Muñoz-Escobar, C. (2015). Coleópteros y otros insectos asociados al páramo magallánico en la región de Magallanes, Chile. En: E. Domínguez y D. VegaValdés (Eds.), Funciones y servicios ecosistémicos de las turberas en Magallanes, Colección Libros INIA $N^{\circ} 33$ (pp. 199-224). Punta Arenas, Instituto de Investigaciones Agropecuarias. Centro Regional de Investigación Kampenaike.

Legoupil, D., \& Sellier, P. (2004). La sepultura de la Cueva Ayayema (Isla Madre de Dios, archipiélagos occidentales de Patagonia. Magallania, 32, 115-124.

Marín, J.C., Varas, V., Vila A., López, R., Orozco-Terwengel, P., \& Corti, P. (2013). Refugia in Patagonian Fjords and the eastern Andes during the Last Glacial Maximum revealed by huemul (Hippocamelus bisulcus) phylogeographical patterns and genetic diversity. Journal of Biogeography, 40, 2285-2296.

Matus, M. (2008). Puerto Edén: El desaliento inesperado del desarrollo. (Tesis de pregrado). Universidad de Chile, Santiago, Chile.

Núñez, E. (2008). Manual para la planificación del Manejo de Áreas Protegidas. Manual Técnico 23. Santiago, Corporación Nacional Forestal.

Naciones Unidas. (2008). Declaración de las Naciones Unidas sobre los Derechos de los Indígenas. Recuperado de http://www.un.org/esa/socdev/unpfii/documents/ DRIPS_es.pdf

Ortiz, J.C. (2015). Anfibios de las turberas del extremo austral de Chile. En E. Domínguez y D. Vega-Valdés (eds.), Funciones y servicios ecosistémicos de las turberas en Magallanes, Colección Libros INIA $N^{\circ} 33$ (pp. 229-240). Punta Arenas, Instituto de Investigaciones 
Agropecuarias. Centro Regional de Investigación Kampenaike.

Pauchard, A., \& Villaroel, P. (2002). Protected Areas in Chile: History, Current Status and Challengues. Natural Areas Journal, 22(4), 318-330.

Riveros, G., Kusch, A., Cárcamo, J., Domínguez, E. (2015). Avifauna en turberas Fuego-Patagónicas. En E. Domínguez y D. Vega (Eds.), Funciones y servicios ecosistémicos de las turberas en Magallanes (pp. 245-275). Punta Arenas: Colección Libros INIA N ${ }^{\circ}$ 33. Instituto de Investigaciones Agropecuarias. Centro Regional de Investigación Kampenaike.

Vela-Ruiz, G. (2009). Contribución desde el enfoque de capitales a la comprensión de la inclusión de comunidades en los procesos generados por áreas protegidas, Región de Magallanes. (Tesis posgrado). Fundación para la Superación de la Pobreza, Piensa un país sin pobreza, Santiago, Chile.

Vela-Ruiz, G., \& Aravena, J. C. (2011). Análisis de propuesta de zonificación de borde costero en sector aledaño al Parque Nacional Bernardo O'Higgins. Punta Arenas, Fundación CEQUA.

Vela-Ruiz, G., Aravena, J. C., \& Torres, J. (2013). Investigación, planificación y estudio del potencial turístico del Parque Nacional Bernardo O'Higgins, Patagonia Chilena. En Actas V Congreso Latinoamericano de investigación turística. Periplo Sustentable, 25, 159-176.

Vela-Ruiz, G., Aravena, J. C., Torres, J., Ruiz, M., Vera-Giusti, J., Huenucoy, C., \& Tonko J.C. (2011). Guía para el desarrollo de proyectos turísticos en el Parque Nacional Bernardo O'Higgins. Punta Arenas, Fundación CEQUA.

Otras fuentes

Ministerio de Desarrollo Social (2014). Decreto 66/2014. Reglamento de Consulta Indígena. Ministerio de Desarrollo Social. 
